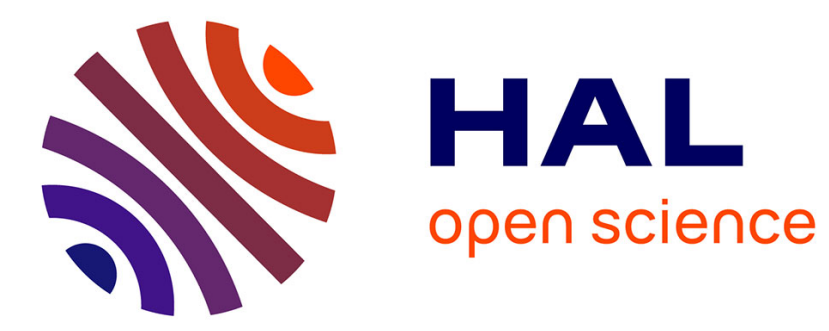

\title{
Ocelocoatl, de l'art secret de communiquer avec les dieux \\ José Contel
}

\section{To cite this version:}

José Contel. Ocelocoatl, de l'art secret de communiquer avec les dieux. Caravelle. Cahiers du monde hispanique et luso-brésilien, 2001, Hommage à Georges Baudot, 76 (1), pp.153-164. 10.3406/carav.2001.1292 . hal-02149240

\section{HAL Id: hal-02149240 \\ https://hal-univ-tlse2.archives-ouvertes.fr/hal-02149240}

Submitted on 6 Jun 2019

HAL is a multi-disciplinary open access archive for the deposit and dissemination of scientific research documents, whether they are published or not. The documents may come from teaching and research institutions in France or abroad, or from public or private research centers.
L'archive ouverte pluridisciplinaire HAL, est destinée au dépôt et à la diffusion de documents scientifiques de niveau recherche, publiés ou non, émanant des établissements d'enseignement et de recherche français ou étrangers, des laboratoires publics ou privés. 


\section{Ocelocoatl, de l'art secret de communiquer avec les dieux} José Contel

Citer ce document / Cite this document :

Contel José. Ocelocoatl, de l'art secret de communiquer avec les dieux. In: Caravelle, n76-77, 2001. Hommage à Georges Baudot. pp. 153-164;

doi : 10.3406/carav.2001.1292

http://www.persee.fr/doc/carav_1147-6753_2001_num_76_1_1292

Document généré le 01/06/2016 


\begin{abstract}
ABSTRACT- Ocelocoatl, "Snake-Jaguar », title given to the quacuilli, the old priest of Tlalloc, is a náhuatl word with a complex symbolism. On the one hand, it's the metaphor of the bag of yauhtli (oceloyataztli) and the censer (coatlemaitl) worn by the priests in the ritual ceremonies celebrated in honour of the gods of rain. On the other hand, it's also that of the awl (Ce-Ocelotl) which made blood spurt out of the veins of the penitents or of those who were sacrified. The blood offering, which was meant of course to obtain rain, was also an act of « gratitude » of men towards Tlalloc.
\end{abstract}

\title{
Resumen
}

RESUMEN- Ocelocoatl, «Serpiente-Jaguar», título que se daba al quacuilli, el viejo sacerdote de Tlálloc, es una palabra náhuatl de simbolismo complejo. Por una parte, es la metáfora de la bolsa de yauhtli \{oceloyataztlî) y del incensario (coatlemaitl) llevados por los sacerdotes en las ceremonias rituales celebradas en honor de las divinidades de la lluvia. Por otra, también lo es del punzón (CeOcelotl) que hacía brotar la sangre de las venas (coacueyeque) de los penitentes o de los sacrificados. La ofrenda de sangre, cuyo objetivo era por supuesto conseguir las lluvias, también era un acto de «reconocimiento» de los hombres para con Tlálloc.

\section{Résumé}

RÉSUMÉ- Ocelocoatl, "Serpent-Jaguar », titre donné au quacuilli, le vieux prêtre de Tlalloc, est un mot nahuatl au symbolisme complexe. D'une part, il est la métaphore de la bourse à yauhtli (oceloyataztli) et de l'encensoir (coatlemaitl) portés par les prêtres dans les cérémonies rituelles célébrées en l'honneur des divinités de la pluie. D'autre part, il l'est aussi du poinçon ( Ce-Ocelotl) qui faisait jaillir le sang des veines (coacueyeque) des pénitents ou des sacrifiés. L'offrande de sang, qui visait bien sûr à obtenir la pluie, était aussi un acte de « reconnaissance » des hommes envers Tlalloc. 


\title{
Ocelocoatl \\ de l'art secret de communiquer avec les dieux
}

\author{
PAR \\ José CONTEL \\ G.R.A.L., Université de Toulouse-Le Mirail
}

Selon le Popol Vuh, à l'aube des temps, après la création du monde, les dieux peuplèrent la terre avec des êtres qui en échange de leur subsistance auraient dû offrir à leurs créateurs offrandes et prières, mais ils en furent incapables: "il ne purent parler en hommes; seulement ils caquetèrent, seulement ils mugirent, seulement ils croassèrent; il ne se manifesta pas de forme de langage, chacun parlant différemment "1. Le courroux des dieux se fit aussitôt sentir. Ils condamnèrent les premiers êtres animés de la terre à être mangés. Devant l'échec de la première création, les dieux décidèrent de façonner des êtres de boue; en vain car, incapables d'adorer leurs créateurs, ils furent détruits. Puis les dieux façonnèrent les hommes de bois mais ceux-ci "n'avaient ni esprit, ni sagesse, nul souvenir de leurs Constructeurs, de leurs Formateurs; sans but ils allaient ils marchaient ${ }^{2}$." Au grand désespoir des dieux, leurs créatures ne pouvaient les nommer. Or, si la parole divine avait coïncidé avec la création du monde, celle des hommes était la condition sine qua non à la reconnaissance des dieux ${ }^{3}$. En effet, les dieux existaient déjà dans l'Autre Monde mais devaient être reconnus pour naître sur terre, parmi leurs créatures. La prière des hommes mais aussi le sacrifice étaient leur nourriture. Réciproquement, l'invocation des forces divines à travers les rites permettait de communiquer avec elles et d'obtenir leurs bonnes grâces.

\footnotetext{
1 Edition et traduction de G. Raynaud, Le Popol Vuh. Les dieux, les héros et les hommes de l'ancien Guatemala d'après le Liure du Conseil, Librairie d'Amérique et d'Orient, 1980, p. 6 .

2 Ibid., p. 9.

3 Ibid., p. 6-7.
} 
Dans les mythes cosmogoniques nahuast, le mécontentement des dieux n'est pas moindre. Les quatre soleils, les ères primordiales, s'achevèrent dans de terribles cataclysmes et ceux qui peuplaient alors la terre furent aussi transformés en animaux, en singes, en poules ou en poissons. Le Soleil de Terre (Tlaltonatiuh) appelé aussi Soleil de Jaguar (Ocelotonatiuh) était peuplé de géants maladroits qui périrent tous dévorés par les jaguars, métamorphoses du dieu Tezcatlipoca, géants dont Tlalloc était le souverain 5 et probablement le créateur 6 .

C'est semble-t-il à cela que font référence les propos du prêtre qui s'adresse au Dieu de la pluie dans le Tlalloc Icuic 7 , "le Chant de Tlalloc ". Dans un premier temps, le prêtre s'identifie à l'un de ces êtres maladroits et s'adresse à son créateur : "si tu fis ce qui est véritablement notre chair", puis il reconnaît l'incapacité des siens à l'adorer correctement : "le premier homme ne te fait que des affronts". A ce mea culpa, Tlalloc répond:

Ahuia canacatella nechyapinvuia anechyyca velmatia, anotata, yno quacuillo ocelocoatlaya.
Ahuia, si quelque part quelqu'un me fait honte, c'est qu'il ne me connaît pas bien, ô mon père mon prêtre au crâne rasé,

Serpent-Jaguar, aya.

Les prêtres et les sorciers, intermédiaires entre les dieux et les hommes, étaient les gardiens et protecteurs de la Nahuallatolli, "parole secrète ", nom que donnaient les nahuas à l'art secret de communiquer avec les dieux ou les forces surnaturelles. Ici, le gardien de la "parole secrète " est le quacuilli ocelocoatl, "le prêtre au crâne rasé, Serpent-Jaguar ", celui qui connaît bien Tlalloc.

\section{Le quacuilli : "le prêtre au crâne rasé "}

Lorsque Tlalloc s'adresse au prêtre, il l'appelle notata "mon père", ceci s'expliquant en partie par le fait que le titre de quacuilli était attribué

4 Cf. par exemple Anales de Cuaubtitlan et Leyenda de los Soles in Códice Chimalpopoca, trad. P. Velázquez, UNAM, México, 1975; Historia de los mexicanos por sus pinturas, in A.M. Garibay, Teogonia e historia. Tres opúsculos del siglo XVI, Porrúa, México, 1985.

5 F.de Alva Ixtlilxóchitl, Obras históricas, Universidad Nacional Autónoma de México (UNAM), México, 1985, I, p. 273.

$6 \mathrm{~J}$. Contel, "Visiones paradisíacas. Extrañas analogías entre Tlallocan y 'Paraíso Terrenal' en la Historia general de las cosas de Nueva España de Fray Bernardino de Sahagún", Fray Bernardino de Sahagún y su tiempo, Universidad de León, León, p. 624-626.

7 Cet hymne à Tlalloc faisait partie du rituel complexe de cérémonies célébrées en son honneur, à Mexico, lors du premier mois de l'année, Atlcahualo (arrêt de l'eau) ou Quauitlehua (le mât est dressé), qui marquait le début de la pénuric d'eau. Cf. J. Contel, Tlalloc: L'Incarnation de la Terre. Naissance et métamorphoses, doctorat 3ème cycle sous la direction de Georges Baudot, Toulouse, 1999, p. 310-333. 
aux prêtres expérimentés ${ }^{8}$. En effet, les sources confirment l'âge avancé des "prêtres tonsurés", par exemple, le Códice Matritense del Real Palacio 9 où il est dit qu'ils étaient chargés de services au temple de Tlalloc et participaient aux rites de Atlcabualo. Les Primeros Memoriales 10 signalent aussi la présence des vieux prêtres de Tlalloc, appelés quacuacuiltin, pour la même fête. Il dirigeaient et mettaient en ordre les gens pendant les processions. Selon Sahagún11, les quacuacuiltin avaient le crâne rasé, ils se distinguaient des autres par le fait qu'ils portaient une longue mèche de cheveux qu'ils laissaient pousser sur la partie supérieure de la tête, à la manière des tlacauhque, les guerriers qui avaient accompli des prouesses au combat 12 .

Ces tlamacazque chargeaient les pénitents (tlamaceuhque), probablement leurs apprentis ou bien des prêtres plus jeunes (teiccatoton) 13 , de porter les offrandes au sommet des montagnes où se trouvaient les statues des divinités. Les vieux sages restaient accroupis devant un feu au milieu de la cour du temple et attendaient le retour du pénitent qui devait suivre scrupuleusement les ordres du prêtre car la moindre infraction autorisait ce dernier à rosser à coups de bâton, voire même à tuer, celui qui avait fauté. Le prêtre était doté d'extraordinaires pouvoirs magiques qui lui permettaient, sans se déplacer, d'observer et suivre à distance tous les faits et gestes du pèlerin. Il s'identifiait à Oxomoco et Cipactonal qui étaient le premier couple d'humains créé par les dieux mais aussi les premiers devins et prêtres 14 . Inventeurs du calendrier, ils incarnaient l'origine des temps et la vieillesse.

8 Cf. Sahagún, Ritos, sacerdotes y atavios de los dioses, trad. M. León-Portilla, UNAM, México, 1958, p. 86-109 et Sahagún, Educación mexica. Antologia de textos sahaguntinos, trad. A. López Austin, UNAM, México, 1985, p. 211 ; Codex de Florence, liv.VI, ch.39, fol.176r-177v in Sahagún, ibid., 1985, p. 61.

9 Códice Matritense del Real Palacio in Sahagún, Historia universal de las cosas de la Nueva España. Codex de Madrid. Bibliothèque du Palais Royal, folios $33 r^{\circ}$ à $129 r^{\circ}$, éd. Et trad. Placer Marey, doctorat 3ème cycle, direction G. Baudot, Toulouse, 1978, fol. 55r ${ }^{\circ}$.

10 Sahagún, Primeros memoriales, ed. facs., University of Oklahoma Press, Norman, 1993, fol. 250 r.

11 Sahagún, Historia General de las cosas de Nueva España. CNCA, Alianza Editorial Mexicana, México, 1989, liv.2, p. 124.

$12 \mathrm{H}$. Ruiz de Alarcón, Tratado de las supersticiones y costumbres gentilicias que hoy viven entre los indios naturales desta Nueva España. Escrito en 1629, SEP, México 1988, p. 4950.

13 Cf. Sahagún, op. cit., 1985, p. 49 et 259.

14 Ruiz de Alarcón, Tratado de las supersticiones..., México, 1988, p. 49-50. 


\section{Ocelocoatl : "Serpent-Jaguar »}

Ocelocoatl, véritable énigme du Tlalloc Icuic, est l'un de ces termes dont l'obscurité - pour reprendre une expression de Georges Baudot 15 à propos des chants sacrés - "a intrigué, et agacé jusqu'à leur compilateur, Fray Bernardino de Sahagún qui y vit l'effet d'une volonté de dissimulation diabolique».

Pour le scholiaste du Tlalloc Icuic, Ocelocoatl doit s'entendre ocelocuaquilli, "le tonsuré à la tête de jaguar ", une forme archaïque de ocelocuaith "tête de jaguar" selon Eduard Seler16. Pour Angel María Garibay 17 , ceci pouvait faire référence à la tonsure des vieux prêtres mais il avoue sa perplexité face à cette version. Si l'interprétation du scholiaste et l'hypothèse des deux érudits s'avéraient exactes, oceloquaitl ou ocelocuaquilli pourrait évoquer certaines représentations iconographiques dans lesquelles Tlalloc est coiffé d'une tête de jaguar18. De nombreux spécialistes ont vu dans l'association serpent-jaguar une évocation des origines olmèques de l'image de Tlalloc19. Bien que ces interprétations ne soient pas à écarter, nous pensons qu'il y en a d'autres.

\section{Ocelocoatl: " le yataztli et le tlemaitl»}

L'interprétation la plus récente, à notre connaissance, est celle de Patrick Saurin ${ }^{20}$ qui analyse une association Jaguar-Serpent relevée dans la Historia General de Sahagún 21. A l'occasion des processions faites en l'honneur des dieux de la pluie, des prêtres arboraient un sac en peau de jaguar (oceloyiataztli), d'où pendaient la queue et les extrémités des quatre pattes de cet animal. Ce sac contenait de l'encens fait d'une poudre d'herbe sèche appelée yaubtli 22. Cette poudre était destinée à être brûlée dans un encensoir appelée tlemaitl ayant l'apparence d'une grande cuillère, avec une tête de serpent sculptée à l'extrémité d'un manche.

L'hypothèse de Saurin est d'autant plus intéressante que le yaubtli (Tagetes Lucida de son nom scientifique) était utilisé en guise d'encens

15 Georges Baudot, Les lettres précolombiennes, Toulouse, Privat, 1976, p. 59-60.

16 Eduard Seler in A.M. Garibay, Veinte himnos sacros de los nabuas, UNAM, México, 1958 , p. 58.

17 Garibay, Veinte himnos sacros..., p. 58.

18 Códice Laud, Fondo de Cultura Económica (FCE), México, 1994, p. 23 ; Códice Vaticano B, FCE, México, 1993, p. 45.

19 J. Contel, Tlalloc ..., op. cit., ch. 1 et 3.

20 P. Saurin, Teocuicatl. C'hants sacrés des anciens Mexicains, Publications scientifiques du Muséum, Paris, p. 65.

21 Sahagún, Historia general de las cosas de Nueva España. Porrúa, México, 1975, p. 147.

22 Ibid., p. 116. 
dans le culte en l'honneur de Tlalloc et des divinités de l'eau'23. Cette herbe aux fleurs jaunes, qui fleurissait en temps de pluie, était la plante de Tlalloc par excellence. On donnait au dieu le nom de Yiaubyo 24 : Seigneur du Yauhtli25. Par ailleurs, l'un des quatre Tlalloque portait le nom de Yauhqueme 26. Elle était un remède contre les maladies de Tlalloc27. Enfin, le Yaubtli est connu pour ses propriétés hallucinogènes. Selon Sahagún, lors de Xocotl Huetzi (10ème fête du xiuhpohualli), on en saupoudrait le visage des captifs " pour qu'ils perdissent le sens et ne sentissent pas trop la mort ${ }^{28}$. "Selon Mercedes de la Garza ${ }^{29}$, les effets psycho-actifs du Tagetes Lucida se produisent par inhalation. Notons enfin que le yauhtli associé à d'autres éléments déjà cités tels que le oceloyataztli, le tlemaitl et le coatl sous forme de bâton ondulé (coatopilli) 30 ou sous forme d'offrande 31 est présent dans la septième Section du compte des destins (tonalpoualli), Ce Quiauith "Un Pluie » dont Tlalloc était le régent 32 .

\section{Ocelocoatl, le poinçon et la veine gorgée de sang}

Selon Ruiz de Alarcón33, dans le langage métaphorique des incantations employé par le saigneur, le nom de coacueyeque (jupes de serpent) était donné aux veines et celui de Tlamacazqui Ce ocelotl (Prêtre ou Pourvoyeur Un-Jaguar) à la lancette servant à les transpercer. Dans

23 Sahagún, op. cit., 1975, Liv.XI, p.688; F. Ortiz, El huracan. Su mitologia y sus simbolos, FCE, México, 1947, p. 289-291.

24 J. Contel, Tlalloc: l'Incarnation de la Terre. Naissance et métamorphoses, Toulouse, 1999 , p. 25.

25 Litt. : "Qui a la qualité du yauhtli" ou " qui est fait de yauhtli".

26 Litr. : "Vêtu de Yaubtli ".

27 Ibid., p. 150.

28 Sahagún, Historia general..., 1989, liv.2, p. 90.

29 Mercedes de la Garza, Le chamanisme nahua et maya. Nagual, rêves, plantes et pouvoir, Guy Trédaniel Editeur, Paris, 1990, p. 127-128.

30 Eléments énumérés et identifiés par J. de Durand-Forest, "Del simbolismo en el Tonalamatl del Códice Borbónicon. The symbolism in the plastic and pictorial representations of Ancient Mexico : a symposium of the 46 CIA (Amsterdam, 1988), Holos, Bonn, 1993, p. 286-289.

31 Selon Jacqueline de Durand-Forest (ibid.), la présence d'un serpent émergeant d'un sac de copal est peut-être une exhortation à faire des offrandes et des sacrifices.

32 Septième treizaine du tonalamatl Ce Quiauitl dont Tlalloc est le régent. in Codex Borbonicus. Manuscrit mexicain de la bibliothèque du Palais Bourbon, Leroux, Paris, 1899 ; Tonalamatl Aubin. Manuscrit Mexicain n 18-19, Bibliothèque Nationale de Paris, éd. Carmen Aguilera, Tlaxcala, 1981 ; Codex Borgia, introduction et commentaires de K. Nowotny, trad. de J. de Durand-Forest, Club du Livre, P. Lebaud Editeur, Paris, 1977.

33 Ruiz de Alarcón, Tratado de las supersticiones..., 1988, p. 199-201. 
certe incantation, le sorcier s'identifie à Tlamacazqui Nabualtecuhtli, c'est-à-dire Tlalloc.

Moi tlamacazqui, je suis le Seigneur Sorcier; je suis à la recherche des quatre têtes (...) je m'adresse à vous mes sœurs aux jupes colorées, jupes de serpent : et toi le Pourvoyeur Un Jaguar, viens car tu vas boire sans retenue jusqu'à la lie...

La même métaphore est présente dans Jacinto de la Serna 34 . Le saigneur (texoxotla) s'adressait aux veines en les appelant serpents (cocoa), puis il parlait à la lancette (ocelotl) et lui demandait de boire le sang jusqu'à la perte de conscience du malade.

La saignée pouvait être aussi pénitentielle et le vocable ocelotl comme métaphore de l'instrument d'autosacrifice évoque les crocs et les griffes redoutables du jaguar, nahualli par excellence des sorciers, mais aussi de certains dieux, tel Tezcatlipoca. Notons que Tezcatlipoca et Tlalloc partageaient le titre de Naualpilli, "Prince Sorcier ". Tlalloc est, en outre, le patron de la septième Section du compte des destins, Ce Quiauitl, signe sous lequel naissaient ceux qui devaient devenir sorciers 35 . Il n'est pas impossible, par conséquent, que l'offrande de sang soit un rite d'actualisation d'Ocelotonatiuh, dont les principaux protagonistes étaient, cela a été dit, Tezcatlipoca et Tlalloc. La mort des géants sous les crocs et les griffes des jaguars était une punition divine infligée à ceux qui ne savaient pas comment s'adresser aux créateurs. A ce propos, il est une expression en nahuatl citée par Olmos 36 qui l'illustre parfaitement :

auh ye nican onotihuitz ycoauh in voici le serpent, le jaguar de Tloque tecuan 37 in tloque nahuaque.

Nabuaque, c'est-à-dire: voici le châtiment du dieu

C'est d'une punition dont il est également question dans Torquemada et Ixtlilxóchitl38. Tous deux témoignent d'un rêve prémonitoire que fit Tezozómoc, le souverain tépanèque, dans lequel Nezahualcoyotl lui apparut en songe une première fois transformé en un aigle qui lui dévorait le cour, puis en un jaguar qui avec ses griffes et ses crocs lui déchiquetait les pieds et se transformait en cœur de l'eau et des

34 Cf. Mercedes de la Garza, Le chamanisme..., op. cit., p .29.

$35 \mathrm{~J}$. Contel, Tlalloc: l'Incarnation de la Terre..., p. 68-71. Pour les liens étroits qui existaient entre Tezcatlipoca et les sorciers et pour les correspondances entre ce dieu, Tlalloc et la pluie voir aussi G. Olivier, Moqueries et métamorphoses d'un dieu aztèque. Tezcatlipoca, le "Seigneur au miroir fumant", CEMCA, Institut d'Ethnologie, Paris, 1997, p. 112-128.

36 Cité par Seler in J. de Durand Forest, "Del simbolismo en el Tonalamatl del Códice Borbónico", op. cit., p. 289.

37 Tecuani signifie en fait "Mangeur d'hommes", nom donné au jaguar mais aussi à d'autres prédatcurs.

38 Torquemada, Monarquia Indiana, Porrúa, México, 1986, I, p.117-118; Alva Ixtlilxóchitl, Obras históricas, UNAM, México, 1985, I, p. 440 et $538 ;$ t. 2 , p. 54. 
montagnes. Ce rêve présageait la fin du tyran tépanèque et de sa lignée mais aussi la destruction de sa cité et de tout son royaume. Ixtlilxochitl39 explique que Tezozomoc, suivant les conseils de ses devins, ordonna à ses fils de tuer à tout prix Nezahualcoyotzin si un jour ils voulaient régner car, faute de quoi,

il (Nezahualcoyotl) retrouverait son empire et boirait leur sang, ainsi l'avaient dit ses devins quand à sa demande ils interprétèrent le rêve de l'aigle et du tigre...

Qu'il fût nabualli d'un roi, d'un dieu, d'un sorcier ou d'un prêtre, ocelotl était donc un tecuani, un "mangeur d'hommes, un buveur de sang ". Il était aussi l'instrument tranchant et aiguisé du texoxotla ou du prêtre pénitent. Dans Ruiz de Alarcón, le poinçon est appelé Ce Ocelotl (Un-Jaguar), qui n'est autre que le signe qui débutait la deuxième treizaine du tonalpohualli. Or, le dieu-patron de cette deuxième période du compte des destins était Quetzalcoatl a qui l'on attribue l'origine de l'autosacrifice. A cet égard, le Codex Vaticanus $A$ et le Codex TellerianoRemensis 40 représentent Quetzalcoatl (Ehecatl) en tant que patron de la deuxième section et un pénitent se transperçant la langue (Fig. 1). Les commentaires du scholiaste du Codex Vaticanus sont riches d'enseignements :

On dit de celui-ci [Quetzalcoatl] qu'il réforma le monde avec la pénitence (...)[II] a été le premier inventeur des sacrifices de sang humain, de toutes les autres choses que l'on offrait aux dieux, et ainsi ils se transperçaient la langue, afin qu'en sorte du sang, ainsi que des oreilles et du membre viril...

Dans le Codex Borgia 41, Quetzalcoatl est représenté en tant que régent du deuxième des vingt signes des jours ( 2 Ehecatl: 2 Vent) et, en exergue, un serpent transpercé d'une flèche qui provoque le jaillissement du sang (Fig. 2). La planche 5 du Codex Laud 42 montre un pénitent nu assis au sommet d'une pyramide. Le poinçon planté dans le dos du personnage et le signe du jour Jaguar évoquent une fois encore l'offrande de sang.

Avant les hommes, les dieux avaient montré l'exemple à Teotihuacan. Nanahuatzin et Tecuziztecatl, le fils de Tlalloc, firent pénitence et offrande de leur sang, puis se jetèrent dans la fournaise divine avant de se métamorphoser en Soleil et en Lune. Il furent respectivement suivis dans leur acte par l'Aigle et le Jaguar 43 .

39 Ixtlilxochitl, ibid, I, p. 440.

40 Códice Vaticano A, FCE, México, 1996, fol.14v et 15r ; Codex Telleriano Remensis, éd. Eloïse Quiñones Keber, University of Texas Press, Austin, 1995, fol. 8v et 9v.

41 Codex Borgia, op. cit., pl. 9.

42 Códice Laud, FCE, México, 1994.

43 Leyenda de los Soles in Baudor, op. cit., p. 76-78. 
Serpent-Jaguar était donc une métaphore de l'offrande de sang destinée à payer la dette aux dieux que l'on voulait honorer. La saignée (nezoliztli) qui provoquait des états altérés de conscience 44 devait avoir aussi pour but d'entrer en contact avec les dieux. Les prêtres communiquaient avec le monde surnaturel à travers l'abstinence, le jeûne, la veille mais aussi l'autosacrifice. Ruiz de Alarcón 45 donne d'intéressantes précisions sur les effets de la saignée sur les prêtres pénitents :

Arrivé où se trouvait l'idole..., il s'inclinait à l'endroit où il devait déposer son offrande. Ceci fait, ils se sacrifiait en faisant couler son sang. Il avait pour cela un poinçon fait avec une tige de roseau pointue... et l'on raconte que certains en perdaient même conscience ou s'endormaient, et dans cette extase, ils entendaient, ou feignaient d'entendre, des voix de leur idole qui leur parlait, ce dont ils étaient très orgueilleux et ils avaient la quasi certitude que ce qu'ils demandaient leur serait accordé...

Les effets de ces pratiques ascétiques étaient comparables à ceux obtenus par l'ingestion de produits hallucinogènes 46 . Ils parvenaient ainsi à un état de transe extatique qui permettait à leur tonalli de s'extérioriser afin d'accéder aux domaines des dieux. Les prêtres du Calmecac attendaient que la nuit fût tombée pour faire pénitence 47 . D'abord, ils se saignaient devant l'image du dieu avec des épines d'agave. Ensuite, ils sortaient nus et se rendaient au sommet d'une montagne pour porter leurs offrandes au dieu qu'ils désiraient honorer.

Pour ces fêtes consacrées à Tlalloc, l'autosacrifice, placé sous le patronage de Quetzalcoatl son inventeur, était aussi important que le sacrifice des enfants. Le quacuilli ocelocoath le vieux prêtre de Tlalloc, était le garant du respect des rites de pénitence, des offrandes de sang en général, car par cet acte magique l'on passait du profane au sacré. Il n'est pas surprenant que le vocable "ocelocoatl" soit utilisé par Cervantes de Salazar 48 pour désigner Quetzalcoat ${ }^{49}$ en tant qu'Ehecatl, le dieu du vent, car celui-ci balayait les chemins pour les dieux de la pluie 50 :

\footnotetext{
44 M.de la Garza, op. cit., p. 29.

45 Ruiz de Alarcón, op. cit., p. 53.

46 M. de la Garza, op. cit., p. 32.

47 Sahagún, Educación mexica..., op. cit., 1985, p. 166-169.

48 Cervantes de Salazar, Crónica de la Nueva España. Madrid, 1914, I, p. 81.

49 Il ne doit pas s'agir d'une erreur de Cervantes, bien qu'on puisse être en droit de le penser dans la mesure où "plume de serpent " n'est pas la traduction d'Ocelocoatl mais plutôt celle de Quetzalcoatl. Le fol.42v. du Codex Tudela décrit l'entité en des termes identiques mais la nomme Quetzalcoatl "... un démon appelé Quetzalcoatl [Quetzatlcouarl], qui veut dire serpent fait de plumes. Les Indiens le tenaient pour dieu du vent... sur la tête on lui mettait une couronne, comme une mitre en peau de tigre... " 50 Sahagún, op. cit., 1989, lib. I, p. 39.
} 
...un démon appelé Ocelocoatl [Oceloocoatl], qui veut dire "plume de serpent ". C'était le dieu du vent... sur la tête on lui mettait une couronne en peau de tigre...

Ocelocoatl, en tant que métaphore de l'offrande de sang, est à rapprocher du Serpent de la Vision des Mayas. En effet, chez ceux-ci la saignée visait à provoquer une vision de serpent dans la gueule duquel apparaissait l'ancêtre ou le dieu avec lequel on voulait entrer en contact. Selon les auteurs de Maya Cosmos 51, le Serpent à Plumes (Kukulkan pour les Maya et Quetzalcoatl en náhuatl) est l'expression de ce concept au Postclassique. Par conséquent, Ocelocoatl (ou Ocelocoatl-Quetzalcoatl) est l'alchimie qui permet au quacuilli de communiquer avec Tlalloc. De la gueule du serpent - notons que le serpent de la vision maya pouvait être bicéphale - devait apparaître le dieu. Le Serpent est le chemin qu'empruntent les dieux pour entrer dans le monde des hommes lorsque ceux-ci les appellent à travers les rites de sang. L'arrivée de Tlalloc sur terre devait être bénéfique et apporter les pluies bienfaisantes, mais elle pouvait aussi être annonciatrice de terribles catastrophes car il était aussi Tetzauhpilli, "le Prince des funestes présages " 52.

Cette association Ocelocoatl-Quetzalcoatl renvoie aux origines mythiques de Tlalloc, rapportées dans l'Histoyre du Mechique 53. Tezcatlipoca et Quetzalcoatl transformés en redoutables serpents descendirent du ciel et déchirèrent le monstre terrestre (Tlaltecuhtli). En fécondant la terre, ils donnèrent vie à Tlalloc, la Première Montagne 54 . Pourquoi ne pas donner alors à la métamorphose ophidienne de Tezcatlipoca le nom d'Ocelocoatl (Serpent-Jaguar) ? Le nahualli le plus courant de Tezcatlipoca n'est-il pas précisément Ocelotl, le jaguar, connu aussi sous le nom de Tepeyolotl, "le Coeur ou Esprit de la Montagne "55? Dans le Popol Vuh, les créateurs de la Terre sont appelés le Cour de la Terre et le Couur du Ciel56. Or, ocelotl en náhuatl est précisément synonyme de tlalli: la terre57. En outre, dans la mesure où coatlsignifie non seulement serpent mais aussi jumeau, Ocelocoatl et Quetzalcoatl

51 Pour le Serpent de la Vision voir Freidel, Schele et Parker, Maya Cosmos. Three thousand years on the shaman's path, New York, p. 207-210.

52 Contel, Tlalloc..., op. cit., 1999, p. 311-314.

53 André Thévet, "Histoyre du Mechique, manuscrit français inédit du XVIème siècle" éd. E. de Jonghe in Journal de la Société des Américanistes Nouvelle série, 1905, vol.2, p. 23-24.

54 Contel, Tlalloc : l'Incarnation de la Terre..., op. cit., p. 46-52.

55 Olivier, op. cit., p. 109-130.

56 Popol Vuh, trad. A. Recinos, FCE, México, 1975, p. 23-24.

57 En effet, Ocelotonatiuh (Soleil de Jaguar) est synonyme de Tlaltonatiub (Soleil de terre). Cf.Olivier, op. cit., p. 118. 
pourraient signifier aussi le Jumeau-Jaguar et le Jumeau-Quetzal (ou Oiseau) ou bien encore le Jumeau de la Terre et le Jumeau du Ciel58.

Le mythe de naissance de Tlalloc est selon nous matérialisée dans l'image du dieu. La statue conservée au Museum von Völkerkunde de Berlin (Fig. 3) en est sans doute la plus pure expression. L'on y voit les corps noués des deux serpents créateurs serrant la tête du dieu comme s'ils l'extirpaient dans sa position fotale des profondeurs de l'utérus de la Terre59.

Dans les cérémonies célébrées en l'honneur de Tlalloc, les offrandes de sang, qui visaient bien sûr à conjurer la sécheresse, actualisaient aussi la naissance de Tlalloc. Quel que fût le moyen employé, les prêtres ou les pénitents accédaient au sacré au moyen de l'extase. Tout acte rituel alimentait la sphère divine, il faisait naître ou re-naître les dieux, lesquels, en retour, alimentaient la vie sur terre. La réciprocité était l'unique garantie de survie de l'Univers. L'acte de création n'est rien sans acte de recréation. Telle est la fonction et le devoir de l'intermédiaire entre les hommes et les dieux. Ocelocoatl, "le Serpent-Jaguar ", est aussi l'alchimie divine qui donna naissance à Tlalloc, il l'est aussi de sa re-con-naissance. Les dieux sont les pères des hommes et réciproquement.

58 Fray Rodrigo García rapporte un mythe cosmogonique mixtèque où il est également question d'une divinité créatrice appelée Serpent-Jaguar. (Culebra de tigre) in A. Caso, Reyes y reinos de la Mixteca, FCE, México, 1977, p. 46.

59 Contel, Tlalloc: l'Incarnation de la Terre..., op. cit., p. 68. 


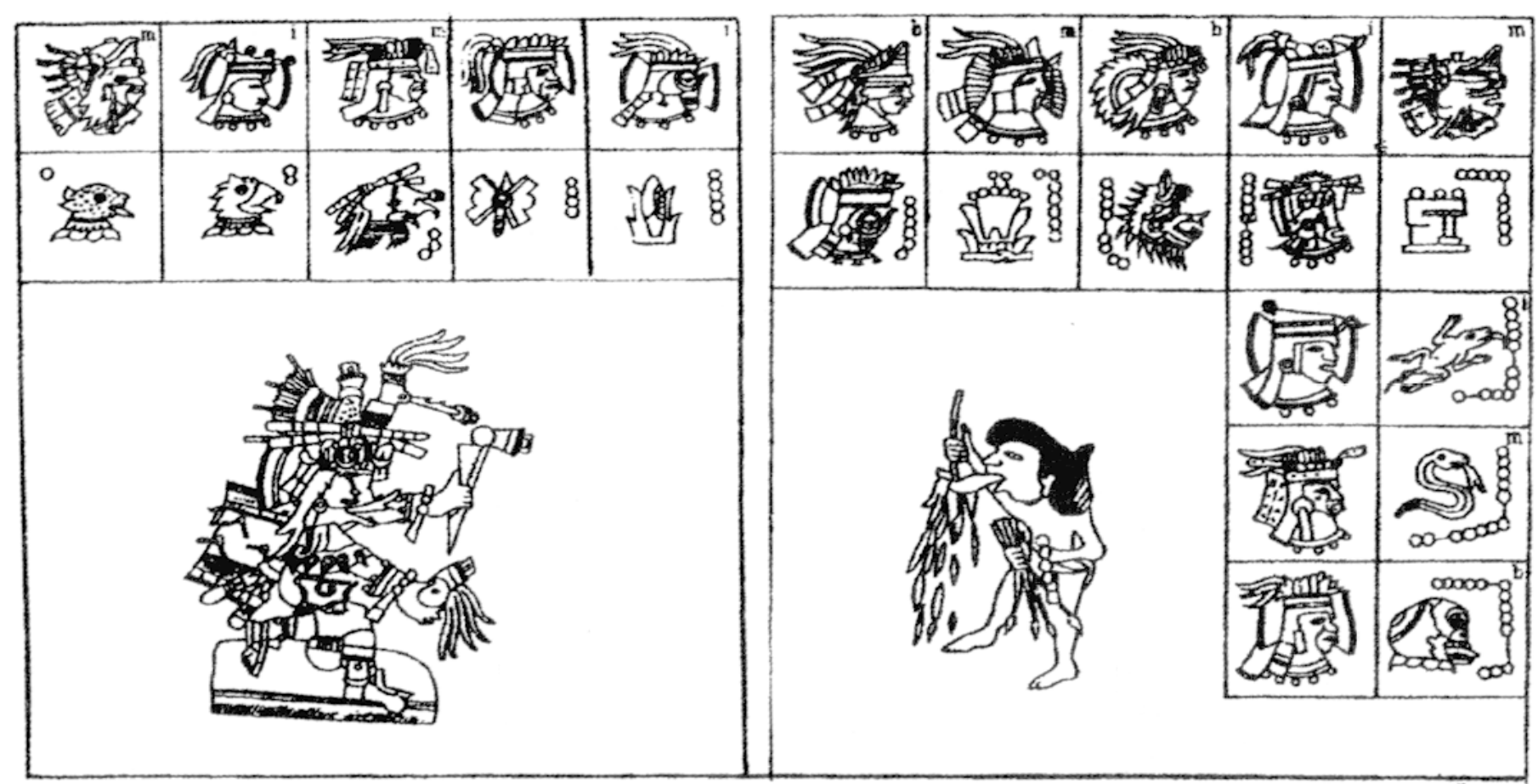

fig. 1 : Quetzalcoatl (Ehecatl), régent de la $2^{\mathrm{c}}$ treizaine Ce Ocelotl : Un-Jaguar, Codex Vaticanus.

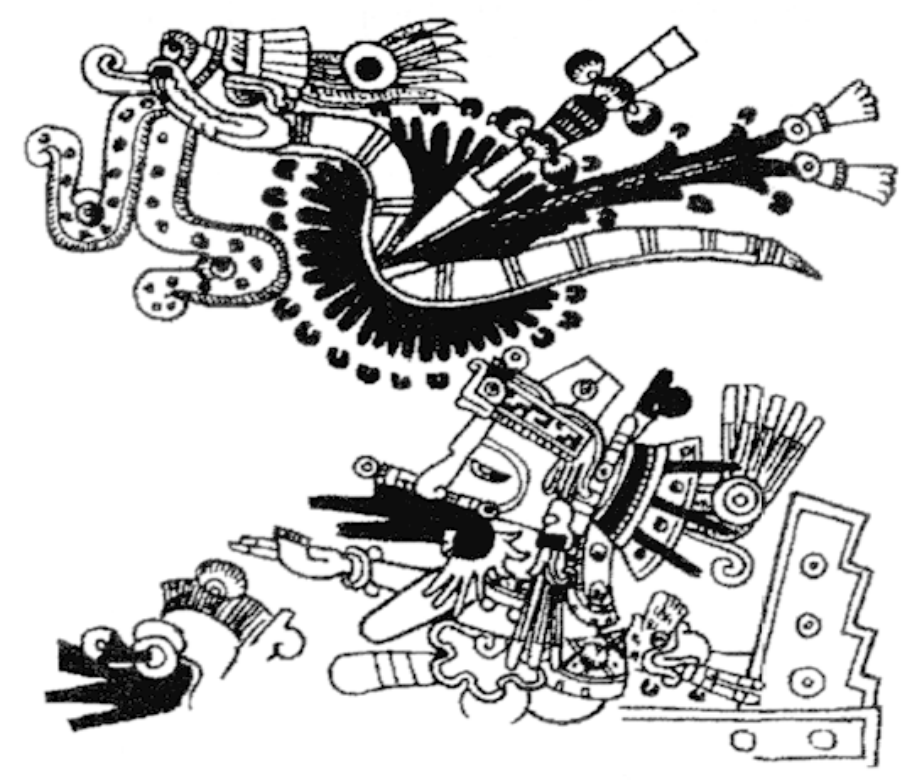

fig. 2 : Le serpent ou la veine gorgée de sang, Codex Borgia, 9.

fig. 3 : Tlalloc, le dieu au visage de serpent, Museum von Völkerkunde, Berlin, (dessins Emilien Contel). 
RÉSUMÉ- Ocelocoatl, "Serpent-Jaguar ", titre donné au quacuilli, le vieux prêtre de Tlalloc, est un mot nahuatl au symbolisme complexe. D'une part, il est la métaphore de la bourse à yaubtli (oceloyataztli) et de l'encensoir (coatlemait) portés par les prêtres dans les cérémonies rituelles célébrées en l'honneur des divinités de la pluie. D'autre part, il l'est aussi du poinçon ( $\mathrm{Ce}$-Ocelotl) qui faisait jaillir le sang des veines (coacueyeque) des pénitents ou des sacrifiés. L'offrande de sang, qui visait bien sûr à obtenir la pluie, était aussi un acte de " reconnaissance " des hommes envers Tlalloc.

RESUMEN-Ocelocoatl, «Serpiente-Jaguar», título que se daba al quacuilli, el viejo sacerdote de Tlálloc, es una palabra náhuatl de simbolismo complejo. Por una parte, es la metáfora de la bolsa de yaubtli (oceloyataztli) y del incensario (coatlemait) llevados por los sacerdotes en las ceremonias rituales celebradas en honor de las divinidades de la lluvia. Por otra, también lo es del punzón ( $C_{e-}$ Ocelot) que hacía brotar la sangre de las venas (coacueyeque) de los penitentes o de los sacrificados. La ofrenda de sangre, cuyo objetivo era por supuesto conseguir las lluvias, también era un acto de "reconocimiento" de los hombres para con Tlálloc.

ABSTRACT - Ocelocoatl, "Snake-Jaguar ", title given to the quacuilli, the old priest of Tlalloc, is a nahuatl word with a complex symbolism. On the one hand, it's the metaphor of the bag of yauhtli (oceloyataztli) and the censer (coatlemaitl) worn by the priests in the ritual ceremonies celebrated in honour of the gods of rain. On the other hand, it's also that of the awl (Ce-Ocelotl) which made blood spurt out of the veins of the penitents or of those who were sacrified. The blood offering, which was mcant of coursc to obtain rain, was also an act of "gratitude " of men towards Tlalloc.

MOTS-CLESS: Mexique central, Période postclassique, Rites religieux, Ocelocoatl, Tlaloc. 